\title{
Anatomical liver segmentectomy 2 for combined hepatocellular carcinoma and cholangiocarcinoma with tumor thrombus in segment 2 portal branch
}

Hiromichi Ishii ${ }^{1 *}$, Takuma Kobayashi', Michihiro Kudou', Masumi Nishimura', Atsushi Toma', Kenji Nakamura', Takeshi Mazaki ${ }^{2}$ and Tsuyoshi Itoh'

\begin{abstract}
Background: Hepatic resection is the only effective treatment for combined hepatocellular carcinoma and cholangiocarcinoma.

Case presentation: A 52-year-old man was preoperatively diagnosed with hepatocellular carcinoma in segment 2 with tumor thrombus in the segment 2 portal branch. Anatomical liver segmentectomy 2, including separation of the hepatic arteries, portal veins, and bile duct, enabled us to remove the tumor and portal thrombus completely. Modified selective hepatic vascular exclusion, which combines extrahepatic control of the left and middle hepatic veins with occlusion of left hemihepatic inflow, was used to reduce blood loss. A pathological examination revealed combined hepatocellular carcinoma and cholangiocarcinoma with tumor thrombus in the segment 2 portal branch. No postoperative liver failure occurred, and remnant liver function was adequate.

Conclusion: The separation method of the hepatic arteries, portal veins, and bile duct is safe and feasible for a liver cancer patient with portal vein tumor thrombus. Modified selective hepatic vascular exclusion was useful to control bleeding during liver transection. Anatomical liver segmentectomy 2 using these procedures should be considered for a patient with a liver tumor located at segment 2 arising from a damaged liver.
\end{abstract}

Keywords: anatomical segmentectomy 2, portal vein tumor thrombus, combined hepatocellular carcinoma and cholangiocarcinoma, modified selective hepatic vascular exclusion

\section{Background}

Combined hepatocellular carcinoma and cholangiocarcinoma (cHCC-CC) is an uncommon primary liver cancer subtype [1] and is difficult to correctly diagnose preoperatively. Most patients with cHCC-CC are preoperatively misdiagnosed with hepatocellular carcinoma (HCC) or cholangiocarcinoma (CC) including our present patient. Hepatic resection leads to improved survival in patients with cHCC-CC [2-5] or HCC with portal vein tumor thrombus (PVTT) [6,7]. In patients with liver cirrhosis, extended liver resection for liver cancer is

\footnotetext{
* Correspondence: ishii0512h@yahoo.co.jp

'Division of Surgery, Kyoto Prefectural Yosanoumi Hospital, 481 Otokoyama, Yosano-cho, Yosa-gun, Kyoto 629-2261, Japan

Full list of author information is available at the end of the article
}

sometimes not feasible because of decreased liver functional reserve; therefore, anatomical segmentectomy or limited non-anatomical hepatectomy must be performed. We herein report an anatomical liver segmentectomy 2 surgical procedure successfully performed for a patient with cHCC-CC and PVTT in the segment 2 portal branch (P2) root arising from liver cirrhosis due to hepatitis B virus. The Brisbane 2000 terminology of liver anatomy and resections was used.

\section{Patient and Method}

A 52-year-old man was admitted to our hospital for treatment of liver tumor. Five years previously, he underwent splenectomy for hypersplenism due to liver cirrhosis, partial gastrectomy for early gastric cancer, and

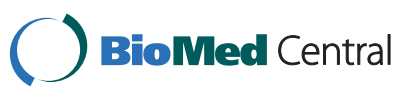

(c) 2012 Ishii et al; licensee BioMed Central Ltd. This is an Open Access article distributed under the terms of the Creative Commons Attribution License (http://creativecommons.org/licenses/by/2.0), which permits unrestricted use, distribution, and reproduction in any medium, provided the original work is properly cited. 
cholecystectomy for a gallstone. Abdominal dynamic computed tomography (CT) revealed a high- and lowdensity lesion in the arterial and venous phases, respectively (Figure 1a and 1b). Although this tumor was fed slightly by the left hepatic artery of segment 3 , the tumor location was liver segment 2 only. The lesion was $4 \mathrm{~cm}$ in diameter and apposed the left hepatic vein. CT also revealed PVTT in the root of P2 (Figure 1c), excluding the umbilical and transverse portion of the left portal vein (Figure 1d and 1e). Based on the CT volumetric study excluding the volume of the tumor, the volumes of total liver, left hemiliver, left lateral section, and segment 2 were 780,351 (45\% of the total liver), 320 (41\%), and $164 \mathrm{~cm}^{3}$ (21\%), respectively. The Child-Pugh classification status and the degree of liver damage scoring system designed by the Liver Cancer Study Group of Japan [8] were determined as class A (5 points) and class A, respectively, based on laboratory data obtained at hospitalization (total bilirubin: $1.2 \mathrm{mg} / \mathrm{dL}$, albumin: $4.5 \mathrm{~g} / \mathrm{dL}$, prothrombin activity: $98.4 \%$, aspartate aminotransferase: $31 \mathrm{IU} / \mathrm{L}$, alanine aminotransferase: $27 \mathrm{IU} / \mathrm{L}$, and indocyanine green retention value at $15 \mathrm{~min}$ after intravenous injection (ICG-R15): 24\%). Considering the impaired liver function indicated by an ICG-R15 of $24 \%$ and the hypertrophied left lateral section, we decided to perform anatomical liver segmentectomy 2 rather than left hepatectomy or left lateral sectionectomy.

\section{Surgical technique}

An upper median skin incision was made, and the round ligament was ligated and divided. We routinely conduct intraoperative ultrasonography for hepatectomy to define the tumor location and vessels to be manipulated for resection. The left lateral section was mobilized by incising the falciform, left coronary, and left triangular ligaments. The left lateral section was retracted to the right, and the Arantius canal was transected on both sides of the umbilical portion of the left portal vein and left hepatic vein. Arantius canal transaction on the side of the left hepatic vein with retraction of the mobilized left lateral section made it easier to encircle the extrahepatic portion of the common trunk of the middle and left hepatic veins. The common bile duct, left portal vein, and left hepatic artery feeding segments 2 and 3 were encircled in the hepatic hilum separately. The round ligament was retracted anteriorly, and the umbilical portion of the left portal vein was exposed by dissecting the serosa of the umbilical fissure. The left hepatic arteries of segment 2 (A2) and 3 (A3) were encircled at the left side of the umbilical portion of the left portal vein after thorough mobilization, and encircling of the umbilical and transverse portions of the left portal vein and P2 by sacrificing one of the portal branches supplying segment 1 (Figure 2a). After A2 was ligated and divided, the liver parenchyma was dissected
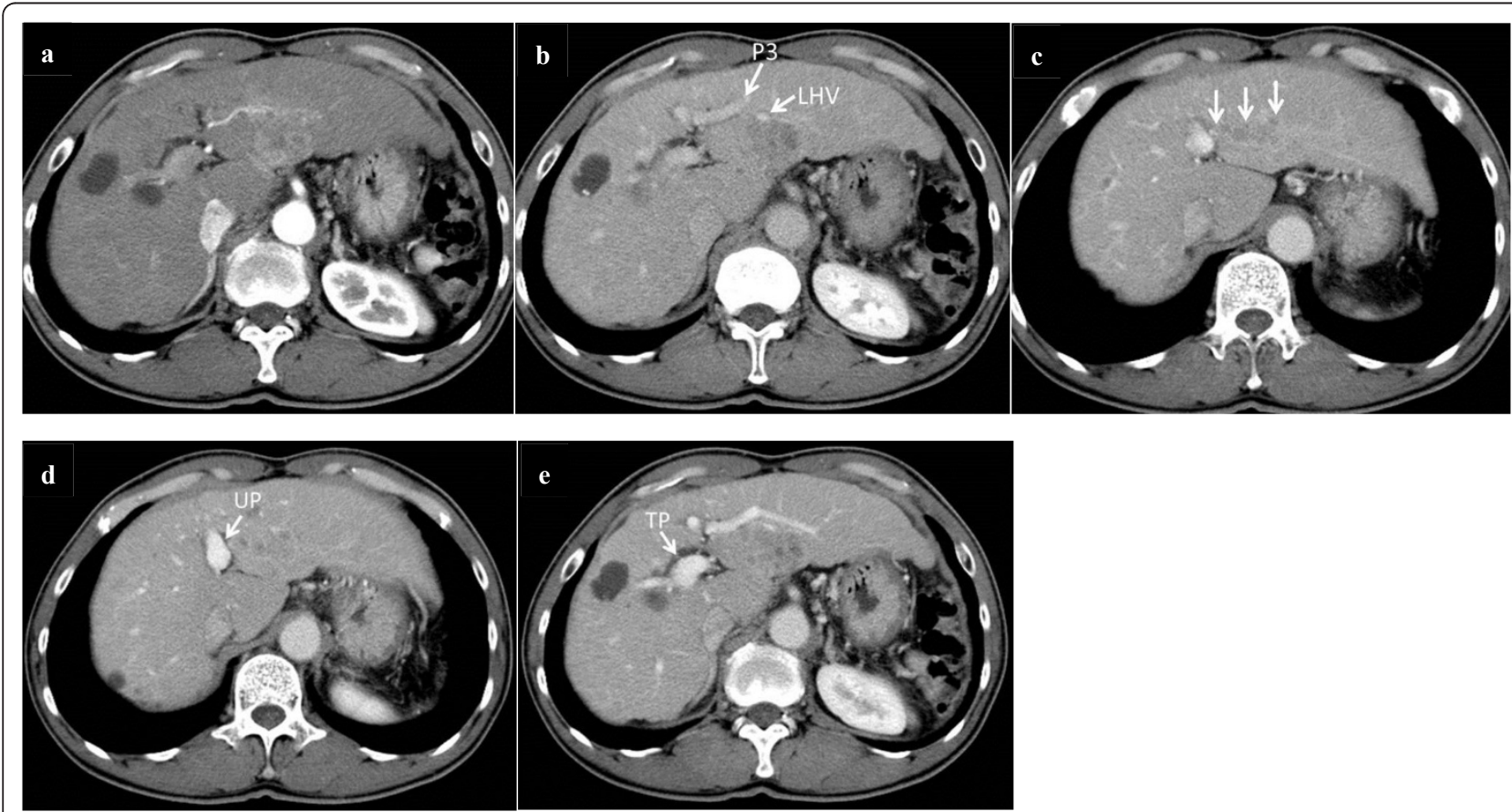

Figure 1 Dynamic computed tomography revealed a tumor in segment 2 of the liver, which was high and low density in the arterial (a) and venous phases (b), respectively, and portal vein tumor thrombus in the portal branch root of segment 2 (arrow) (c). Portal vein tumor thrombus did not invade to umbilical and transverse portions (UP and TP) of the left portal vein (d, e). P3, portal branch of segment 3; LHV, left hepatic vein. 


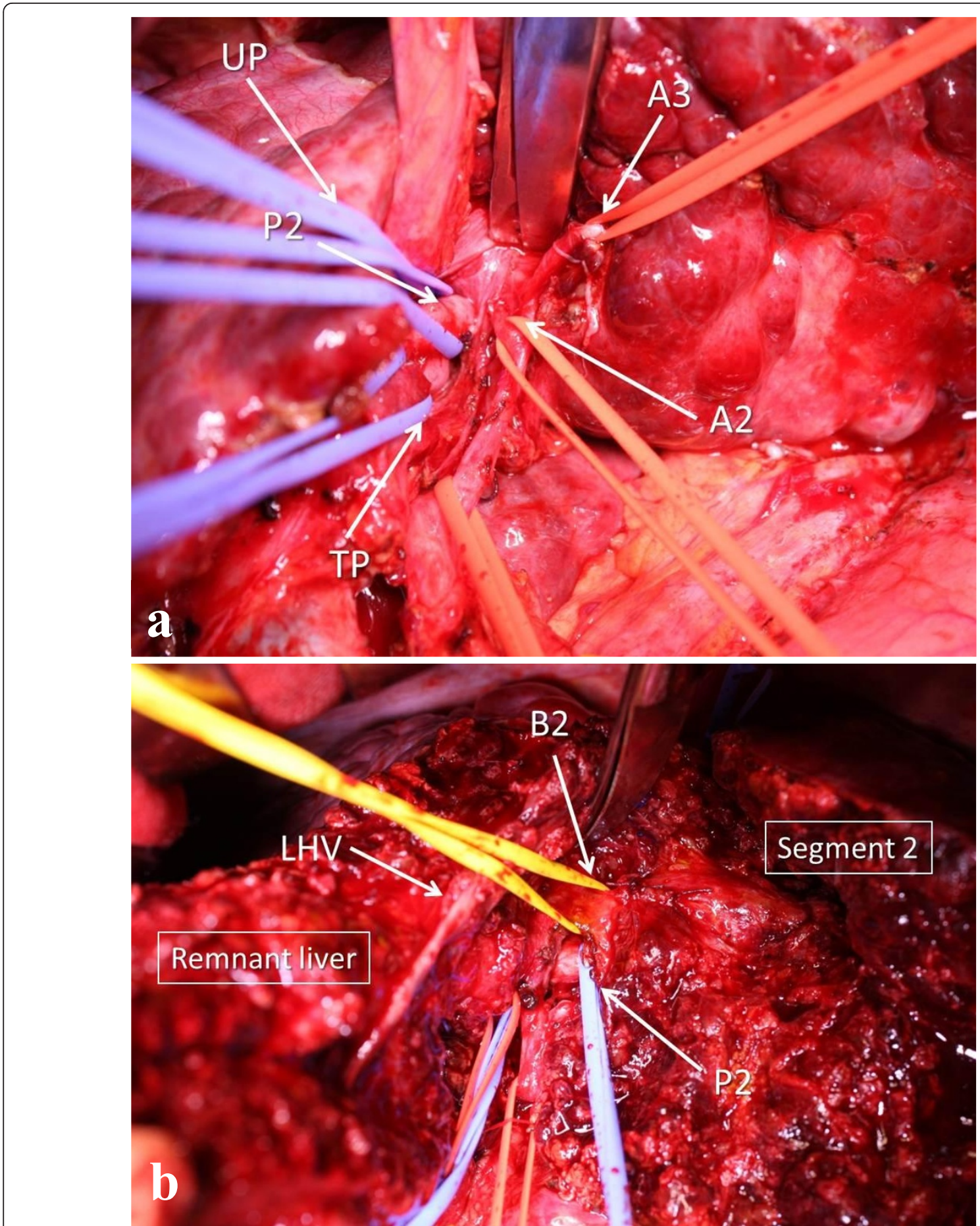

Figure 2 Left hepatic arteries of segment 2 (A2) and 3 (A3), umbilical and transverse portions (UP and TP) of the left portal vein, and portal branch of segment 2 (P2) were encircled (a). Segment 2 and remnant liver were connected only through the portal branch and bile duct of segment 2 (P2 and B2) (b). LHA, left hepatic artery. LHV, left hepatic vein.

using the Cavitron Ultrasonic Surgical Aspirator $\left(\right.$ CUSA $\left.^{\circledR}\right)$ under left hemihepatic vascular occlusion [9] from the periphery toward the vena cava along the demarcation line separating segments 2 and 3 that appeared by clamping the umbilical portion of the left portal vein and A3. Bleeding from the left hepatic vein during transection was controlled using an extrahepatic clamp on the common trunk of the middle and left 
hepatic veins. After liver transection, segment 2 and the remnant liver were connected through only P2 and the segment 2 bile duct (B2) (Figure 2b). Following ligation and division of the B2, P2 was incised at its origin after clamping the umbilical and transverse portions of the left portal vein to ensure complete removal of the tumor thrombus under direct vision. The P2 stump was then closed with continuous sutures. The cut surface of the liver with exposure of the left hepatic vein, indicating the anatomical landmark dividing segments 2 and 3.

Operative and left hemihepatic vascular occlusion times were 419 and $92 \mathrm{~min}$, and blood loss during the operation and liver dissection were 939 and $500 \mathrm{ml}$. No blood transfusions were required during or after operation.

The postoperative course was good, with peak levels of serum aspartate aminotransferase, alanine aminotransferase, and total bilirubin of $264 \mathrm{IU} / \mathrm{L}, 243 \mathrm{IU} / \mathrm{L}$, and $1.6 \mathrm{mg} / \mathrm{dl}$. A left portal vein thrombus was revealed by $\mathrm{CT}$ and ultrasonography on postoperative day 8 ; however, this thrombus disappeared with anti-coagulant therapy. He was discharged 17 days after surgery.

Macroscopically, PVTT was found in P2, and the surgical margin of PVTT was $1 \mathrm{~mm}$ (Figure 3a). Microscopically, the tumor was composed of HCC with trabecular structure and adenocarcinoma (Figure 3b), suggesting $\mathrm{cHCC}-\mathrm{CC}$. The patient is alive 9 months after surgery, and has been undergoing systemic chemotherapy using orally administered S-1 for multiple lymph nodes metastasis.

\section{Discussion}

The World Health Organization [10] defined cHCC-CC as a rare tumor containing unequivocal elements of intimately admixed HCC and CC. This tumor is distinguished from separate $\mathrm{HCC}$ and $\mathrm{CC}$ arising within the same liver. Patients with $\mathrm{CHCC}-\mathrm{CC}$ and $\mathrm{HCC}$ are clinicopathologically similar in average age at the time of diagnosis (i.e., 50-60 years old), with a male predominance, viral hepatitis, elevated $\alpha$-fetoprotein, and liver cirrhosis $[3,4,11]$, and clinicopathological features in our case were similar to the above data. It is difficult to make a differential diagnosis of $\mathrm{CHCC}-\mathrm{CC}$ or $\mathrm{HCC}$; however, therapeutic options for patients with these tumors are almost the same, and liver resection leads to improved survival for both tumors [2-7].

Because a liver tumor located in segment 2 with PVTT in P2 is thought to readily metastasize through the portal venous flow to segments 3 and 4, left hepatectomy is the first choice of treatment. Left lateral sectionectomy is the second choice because the tumor was close to the left hepatic vein. However, in patients with liver cirrhosis, the left lateral section hypertrophies, and left hepatectomy or left lateral sectionectomy are sometimes not feasible because of decreased liver functional reserve and the relatively large volume of the left hemiliver or left lateral section [12]. In our case, the left hemiliver and left lateral section became large (45\% and $41 \%$ of the total liver, respectively) and the ICG-R15 level was relatively high (24\%). And we judged preoperatively that we can exfoliate the tumor from the left hepatic vein. Therefore, anatomical liver segmentectomy 2 rather than left hepatectomy or left lateral sectionectomy was selected because it optimizes the balance between oncological requirements and the need to spare functioning liver parenchyma. Although preoperative surgical planning was evaluated by two-dimensional CT in our case, we think that three-dimensional CT computer-assisted preoperative surgical planning may be helpful for anatomical liver segmentectomy 2 [13]. In anatomical segmentectomy 2, transection of the glissonean pedicle that feeds segment 2 [12] and intraoperative ultrasound-guided blunt compression of the segment 2 portal branch [14] have been reported; however, these techniques are not suitable in liver cancer patients with PVTT in the root of P2, including our patient. In such cases, complete removal of PVTT is extremely important to prevent early tumor recurrence; therefore, after A2, A3, P2, and umbilical and transverse portions of the left portal vein were separated, the liver was dissected, and PVTT was removed under direct vision. It is easy to ligate and divide the origin of P2 before liver transection; however, we think that it is safer and more certain to incise the origin of P2 and suture the stump of P2 after liver transection than before liver transection. This separation method could be adapted for segmentectomy 3 and segmentectomy 3 and 4 [15]. Although several reports [16,17] have demonstrated the feasibility and safety of laparoscopic anatomic resection based on three-dimensional CT images recently, we performed anatomical liver segmentectomy 2 by open surgery because laparoscopic approach to hepatic tumors remains a challenge, and the patient in present report underwent upper abdominal surgeries (splenectomy, partial gastrectomy, and cholecystectomy). Left hemihepatic vascular occlusion, limited to $30 \mathrm{~min}$ followed by $5 \mathrm{~min}$ of perfusion, is useful to prevent blood loss originating from hepatic inflow [9]; however, it is difficult to control retrograde bleeding from the left hepatic vein using this maneuver. Selective hepatic vascular exclusion (SHVE), which combines inflow vascular occlusion (Pringle maneuver) with extrahepatic control of the major hepatic veins, overcomes the drawbacks of backflow bleeding of the Pringle maneuver [18]. In segmentectomy 2 or 3 , modified selective hepatic vascular exclusion (m-SHVE), which combines extrahepatic control of the middle and left hepatic veins with left hemihepatic inflow occlusion, is sufficient to reduce both backflow and inflow bleeding. In our case, this m-SHVE procedure contributed to reduce bleeding during liver 

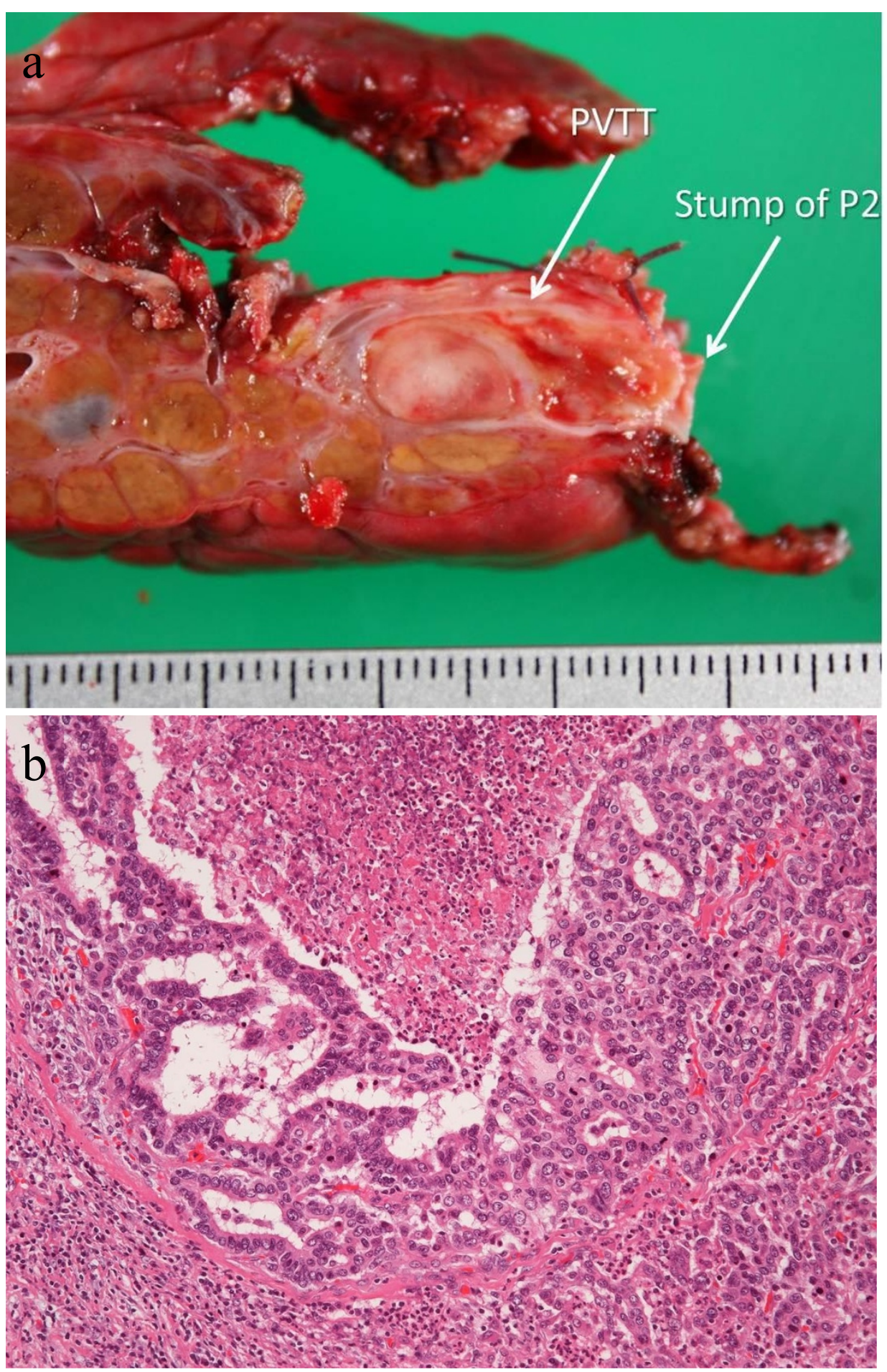

Figure 3 Portal vein tumor thrombus (PVTT) in the segment 2 portal branch (P2) (a). Microscopically, the tumor was combined hepatocellular carcinoma and cholangiocarcinoma (Hematoxylin and eosin staining; magnification $\times 10)(b)$.

dissection and was not associated with hemodynamic changes. SHVE has been used in major liver resections to control intraoperative bleeding $[19,20]$; however, this technique has not been reported and studied in anatomical liver segmentectomy 2, to our knowledge. Furthermore, although it is reported in previous publications that SHVE entails Pringle maneuver and extrahepaic clamping of major hepatic veins $[19,20], \mathrm{m}$-SHVE 
which entails not Pringle maneuver but hemihepatic inflow occlusion has not been reported. Therefore, anatomical liver segmentectomy 2 with m-SHVE is the novel technique. We think that this $\mathrm{m}$-SHVE is the effective technique in anatomical liver segmentectomy 2 and can be adapted also for anatomical liver segmentectomy 3 . Intraabdominal adhesion resulting from previous surgery was very severe, and then, about $120 \mathrm{~min}$ were required and blood loss was about $400 \mathrm{ml}$ during exfoliation of this adhesion. Therefore, we think that the operative time (419 $\mathrm{min})$ and the amount of blood loss $(939 \mathrm{ml})$ in our case seem in the tolerance. The liver transection time was relatively longer because of the hard texture of liver parenchyma resulting from liver cirrhosis. Because postoperative liver function was adequate in our case, anatomical segmentectomy 2 is feasible to preserve remnant liver function in selected patients with liver cirrhosis.

Survival of patients with $\mathrm{cHCC}-\mathrm{CC}$ was significantly poorer than that of $\mathrm{HCC}$ or CC patients [3-5], and the PVTT was found to be significant predictor of poor outcome [3,5]. In our case, the patient is alive 9 months after surgery with lymph nodes metastasis. Although limited hepatectomy was performed, the patient has no recurrence in the remnant liver. Therefore, in selected patients with liver cirrhosis, limited hepetectomy including anatomical segmentectomy 2 may be an appropriate operation even if patients have the PVTT.

\section{Conclusions}

The hepatic arteries, portal veins, and bile duct separation method is a useful approach for a liver cancer patient with PVTT, and m-SHVE is effective for minimizing bleeding during liver resection of segmentectomy 2. Anatomical liver segmentectomy 2 contributes to preserve remnant liver function; however, further investigation is needed to evaluate the overall survival rates in patients who undergo segmentectomy 2.

\section{Consent}

Written informed consent was obtained from the patient for publication of his clinical details and accompanying images. A copy of the written consent is available for review by the Editor of this journal.

\footnotetext{
Author details

${ }^{1}$ Division of Surgery, Kyoto Prefectural Yosanoumi Hospital, 481 Otokoyama, Yosano-cho, Yosa-gun, Kyoto 629-2261, Japan. Division of Pathology, Kyoto Prefectural Yosanoumi Hospital, 481 Otokoyama, Yosano-cho, Yosa-gun, Kyoto 629-2261, Japan.
}

\section{Authors' contributions}

$\mathrm{HI}$ wrote the first draft of this report. HI, MN, and TI performed the operation. TM performed the pathological examination. $\mathrm{HI}$ is the guarantor of the paper. All authors read and approved the final manuscript.

\section{Competing interests}

The authors declare that they have no competing interests.

Received: 9 September 2011 Accepted: 25 January 2012

Published: 25 January 2012

\section{References}

1. Goodman ZD, Ishak KG, Langloss JM: Combined hepatocellular cholangiocarcinoma. A histologic and immunohistochemical study. Cancer 1985, 55:124-135.

2. Jarnagin WR, Weber S, Tickoo SK, Koea JB, Obiekwe S, Fong Y, DeMatteo RP, Blumgart LH, Klimstra D: Combined hepatocellular and cholangiocarcinoma: demographic, clinical, and prognostic factors. Cancer 2002, 94:2040-2046.

3. Yano $Y$, Yamamoto J, Kosuge T, Sakamoto $Y$, Yamasaki S, Shimada K, Ojima H, Sakamoto M, Takayama T, Makuuchi M: Combined hepatocellular and cholangiocarcinoma: a clinicopathologic study of 26 resected cases. Jpn J Clin Oncol 2003, 33:283-287.

4. Liu CL, Fan ST, Lo CM, Ng IO, Lam CM, Poon RT, Wong J: Hepatic resection for combined hepatocellular and cholangiocarcinoma. Arch Surg 2003, 138:86-90

5. Park H, Choi KH, Choi SB, Choi JW, Kim do Y, Ahn SH, Kim KS, Choi JS, Han KH, Chon CY, Park JY: Clinicopathological characteristics in combined hepatocellular-cholangiocarcinoma: a single center study in Korea. Yonsei Med J 2011, 52:753-760.

6. Lin DX, Zhang QY, Li X, Ye QY, Lin F, Li LL: An aggressive approach leads to improved survival in hepatocellular carcinoma patients with portal vein tumor thrombus. J Cancer Res Clin Oncol 2011, 137:139-149.

7. Minagawa M, Makuuchi M, Takayama T, Ohtomo K: Selection criteria for hepatectomy in patients with hepatocellular carcinoma and portal vein tumor thrombus. Ann Surg 2001, 233:379-384.

8. Liver Cancer Study Group of Japan: The general rules for the clinical and pathological study of primary liver cancer. 2nd English edition. Tokyo: Kanehara Press; 2003.

9. Makuuchi M, Mori T, Gunven P, Yamazaki S, Hasegawa H: Safety of hemihepatic vascular occlusion during resection of the liver. Surg Gynecol Obstet 1987, 164:155-158.

10. Wittekind C, Fischer HP, Ponchon T: Combined hepatocellular and cholangiocarcinoma. In World Health Organization classification of tumours of the digestive system. Edited by: Hamilton SR, Aaltonen LA. Lyon: IARC Press; 2000:181.

11. Kim JH, Yoon HK, Ko GY, Gwon DI, Jang CS, Song HY, Shin JH, Sung KB: Nonresectable combined hepatocellular carcinoma and cholangiocarcinoma: analysis of the response and prognostic factors after transcatheter arterial chemoembolization. Radiology 2010, 255:270-277.

12. Sadamori H, Matsuda H, Shinoura S, Umeda Y, Yoshida R, Sato D, Utsumi H, Ohnishi $H$, Yagi T: Anatomical subsegmentectomy in the lateral segment for hepatocellular carcinoma. Hepatogastroenterology 2009, 56:1511-1515.

13. Radtke A, Sotiropoulos GC, Molmenti EP, Schroeder T, Peitgen $\mathrm{HO}$, Frilling A, Broering DC, Broelsch CE, Malago' M: Computer-assisted surgery planning for complex liver resections: When is it helpful? A single-center experience over an 8-year period. Ann Surg 2010, 252:876-883.

14. Torzilli G, Makuuchi M: Ultrasound-guided finger compression in liver subsegmentectomy for hepatocellular carcinoma. Surg Endosc 2004, 18:136-139.

15. Kawasaki S, Makuuchi M, Harada H, Takayama T, Kosuge T: A new alternative hepatectomy method for resection of segment 3 and 4 of the liver. Surg Gynecol Obstet 1992, 175:267-269.

16. Mutter D, Dallemagne B, Bailey Ch, Soler L, Marescaux J: 3D virtual reality and selective vascular control for laparoscopic left hepatic lobectomy. Surg Endosc 2009, 23:432-435.

17. Ueno S, Sakoda M, Kurahara H, lino S, Minami K, Ando K, Mataki Y, Maemura K, Ishigami S, Takumi K, Fukukura Y, Natsugoe S: Preoperative segmentation of the liver, based on 3D CT images, facilitates laparoscopic anatomic hepatic resection for small nodular hepatocellular carcinoma in patients with cirrhosis. Hepatogastroenterology 2010, 57:807-812.

18. Lau WY, Lai ECH, Lau SHY: Methods of vascular control technique during liver resection: a comprehensive review. Hepatobiliary Pancreat Dis Int 2010, 9:473-481. 
19. Fu SY, Lai EC, Li AJ, Pan ZY, Yang Y, Sun YM, Lau WY, Wu MC, Zhou WP: Liver resection with selective hepatic vascular exclusion: a cohort study. Ann Surg 2009, 249:624-627.

20. Zhou W, Li A, Pan Z, Fu S, Yang Y, Tang L, Hou Z, Wu M: Selective hepatic vascular exclusion and Pringle maneuver: a comparative study in liver resection. Eur J Surg Oncol 2008, 34:49-54.

doi:10.1186/1477-7819-10-22

Cite this article as: Ishii et al:: Anatomical liver segmentectomy 2 for combined hepatocellular carcinoma and cholangiocarcinoma with tumor thrombus in segment 2 portal branch. World Journal of Surgical Oncology 2012 10:22.

Submit your next manuscript to BioMed Central and take full advantage of:

- Convenient online submission

- Thorough peer review

- No space constraints or color figure charges

- Immediate publication on acceptance

- Inclusion in PubMed, CAS, Scopus and Google Scholar

- Research which is freely available for redistribution

Submit your manuscript at www.biomedcentral.com/submit
() Biomed Central 